\title{
Stakeholder perception of the ethics of an industry: The case of organic food in South India
}

\author{
By Jubin Jacob-John ${ }^{1}$, Navin Veerapa ${ }^{1}$
}

\begin{abstract}
Increasing reliance on artificial and non-sustainable methods of agriculture had resulted in negatively affecting both the soil and the health of agriculturists. Organic agriculture abhors such conventional agricultural practices and inculcates the implementation of safer, socially responsible and environmentally sustainable agricultural practices. This paper analyses the stakeholders' perception of responsibility and environmental sustainability by conducting qualitative research using case studies of several organic food supply chains based in Kerala, a state in the south of India. The interviews with key stakeholders along the supply chain network illustrate that the rationale for organic agriculture is based on principles of fairness, responsibility and sustainability. Furthermore, the results depict that perceptions of ethics vary amongst stakeholder groups. Thoughthe literature is extensive on business ethics and sustainability, research on stakeholder perception along the entire industry is minimal within academic literature and the paper contributes to this area. Furthermore, from a managerial perspective, this paper sheds light on the influence of business ethics within an industry and exhibits the importance and influence of social responsibility and environmental sustainability within an industry.
\end{abstract}

Keywords: Ethics, food, sustainability, responsibility, agriculture, organic

\section{Introduction}

From paddock to plate is an arduous task due to the involvement of a plethora of actors within the global Food Supply Chain (FSC) context. This has resulted in several transparency and traceability related issues, thereby culminating in the preponderance of unethical, non-sustainable and socially irresponsible behaviour by some actors along the supply chain. The organic produce industry is widely revered as a more responsible form of agriculture and as Stolze and Lampkin (2009)states, organic farming is based onenvironmental sustainability,, social welfare and higher quality food. The principles of organic farming, as defined by the International Federation of Organic Agricultural Movements, is a testament for such environmental and societal underpinnings.

The southern state of Kerala in India is a state with a high percentage of literacy. Stakeholders considers the state an anomaly within India due to its reliance on food from neighbouring states, though the state has abundant rainfall and nutrient rich soil suitable for farming. However, due to decreased labour supply, cultivation within Kerala is predominantly perennial tree crops and seasonal crops(Jeromi, 2007). As a result, cultivation of black pepper, coffee, tea and spices are the predominant agricultural crops 
existent in Kerala.With a higher level of education and understanding of the adverse health effects of employing artificial fertilizers and pesticides, the FSC stakeholders have a pro-organic attitude. As illustrated by Chandrashekar (2010), a large number of farmers in Kerala forayed into organic agriculture and the reason for this could be the sustainability and social inclination of these agriculturists. This paper analyses the reason for this shift into organic agriculture and studies the ethical perceptions of stakeholders involved within the organic food supply chain in Kerala.

Currently, food management involves the coordinated efforts of several actors within the FSC network and this usually transcends national boundaries of trade. The organic food industry focussed in this research involved the primary production within India and then a majority of the product is for export. Since the industry involved several actors, who are business stakeholders, the stakeholder's perception of ethics could differ based on the relative position within the supply chain and their function or role. For the case of simplicity and rigour of the research process, the authors divided these supply chain stakeholders into four groups who have linear relationships with each other and then, the paper illustrates the difference in ethical perception within these stakeholder groups and illustrates explanations for such a difference.

\section{Literature review}

The International Federation of Organic Agricultural Movements (IFOAM) defines four principles that the organic food industry have to account for and this includes the principles of health, ecology, fairness and care. The ethical perceptions of the organic industry have its ethical underpinning on these principles of organic agriculture (Freyer, Bingen, Klimek, \& Paxton, 2015). The principle of health relates to not only the health of the plants, but also the health of animals, human beings and the planter in general. The principle of ecology mandates the agriculture system to work in tandem with the natural ecological systems and cycles by emulating the natural ecological systems and sustaining them. The third principle, the principle of fairness, mandates the nonopportunistic conduction of business by operating in a respectful, equitable and fair manner to all stakeholders within the industry. The principle of care implies that the operations of the business must be responsible to all stakeholders and this should sustain the well-being of both the current and the future generations. As these principles of the IFOAM illustrates, the core values of the organic agricultural industry have its base on sustainability and social responsibility and this is also illustrated by Glasbergen (2011) by exemplifying IFOAM's activities. Ideally, these values will permeate the entire industry. Therefore, such values and value centric operations will be adhered by all stakeholders. Within this research context, this research analyses if these values affect the ethics of the organic industryby understanding the ethical perceptions of all the supply chain actors.

Sligh and Cierpka (2007) define the concept of organic farming as a way of producing food products without artificial means, thereby implying non-usage of artificial fertilisers and pesticides. They define organic agriculture as a system of agriculture aimed at enhancing the agro-ecosystem health by employing agronomic, biological and mechanical methods of farming rather than synthetic materials. Similarly, Gadzikwa, Lyne, and Hendriks (2006)'s definition of organic agriculture encompasses the sustainability 
ideology by defining organic agriculture as a mode of agriculture which has the farming cycles based on biological and natural cycles of the environment and minimising use of off-farm inputs for agriculture. In the initial years of organic agriculture,this was defined as a system of food production that is environmentally sustainable (Stockdale et al., 2001). These definitions did not account for the social implications of the industry. In recent times, as Sligh and Cierpka (2007) illustrates, the accountability and fairness of the industry are being prioritised to the same degree as that of the environmental stewardship of organic agriculture; thereby accounting for the social aspect of this industry. As Lamine and Bellon (2009) and Darnhofer, Lindenthal, Bartel-Kratochvil, and Zollitsch (2010) suggests, the organic industry is undergoing conventionalisation; a process wherein the organic industry's businesses comply with regulations pertaining to organic agriculture but not to the principles of organic agriculture. Klonsky (2012) exemplifies this by citing the case of organic agriculture being permitted to use some specific synthetic materials for agriculture and this, as Klonsky (2012) states, is adverse to the organic ideology of not using artificial man-made inputs. This implies that ethical values pertaining to the environmental sustainability and the responsibility to the society is, today, not a factor affecting organic FSC networks and the players within this industry. The paper aims to analyse if the conventionalisation has percolated the organic industry within Kerala by analysing the ethical perceptions of supply chain actors along the supply chain.

Research of values and ethical underpinning of different stakeholders of the organic food industry by the likes of Duram (1999), Lea and Worsley (2005), De Wit and Verhoog (2007); Honkanen, Verplanken, and Olsen (2006)and Zander and Hamm (2010) illustrates that there is a difference in preference for ethical attributes and value perception among different stakeholder groups. This paper analyses difference in priority associated with different ethical attributes including environmental sustainability and social responsibility. Using a qualitative methodology, the researchers aim to analyse if there is a difference between actors within the supply chain and potential reasons for this. Macharia, Collins, and Sun (2013) shows that in comparison to the developed world, the developing world's FSC is currently, marred by the incessant use of pesticides, ineffective treatment of water, incompetent market systems and unfair remuneration to agriculturists. Since the ethical perceptions of the farmer, trader and such supply chain actors affect this industry's sustainability and social responsibility, a study of such ethical perceptions of these business stakeholders will shed light into the ethical underpinning of the organic food supply chains in the developing world.

\section{Methods}

To understand the perception of ethics held by stakeholders within the organic food industry of Kerala, it was imperative that researchers employ a qualitative research methodology since the researchers wanted an in-depth understanding of behaviour associated with ethical conduction of business. As Zainal (2007) suggests, the case study methodology "allows the exploration and understanding of complex issues" and since the research studied the ethical underpinning of a stakeholder, which was a complex phenomenon in itself, the authors considered a case study approach. Therefore, 
conducted a qualitative research methodology involving multiple detailed cases of organic fresh produce supply chains (in line with the multiple case study approach). Filipe, Santos, and Eisenhardt (2004) illustrates the multiple case study methodology as a variant of the case study methodology, wherein there are multiple observations of the same phenomenon and thereby increase generalizability of the findings. For this, the authors studied multiple supply chains and their stakeholders. This involved the study of entire supply chains since pilot interviews illustrated that the ethical underpinning of stakeholders differed based on their relative role and position within the food value chain. Such a research method will generate more robust and generalizable theory in comparison to single case study approach.

The authors divided the supply chain actors into four groups based on their roles within the organic food supply chain. Since the definition of the 'organic' produce was not clear and certification systems were inefficient within the context research, the researchers conducted the research on non-conventional FSC actors. This non-conventional FSC actors included actors involved in the farming and trade of certified organic products, natural produce, and produce farmed through 'Good Agricultural Practices' (GAP). These actors were selected since they were involved in the trade of products that, though not certified organic, were produced in a more sustainable and ethical manner. The principal researcher contacted some of these supply chain actors via telephone, emails and social media and once the authors identified members within a supply chain, they identified then the other actors within the supply chain through snowball sampling (chain referral). To reduce biases and gain meaningful data the authors sorted, the interviewed actors based on their turnover, which means that, alternatively, the researchers interviewed both large and small players within each supply chain.

\section{Findings}

Consumers perceived the produce consumed in Kerala, which was cultivated in neighbouring states, to have a higher degree of pesticide concentration and this has resulted in an increase in consumers within Kerala mandating safer foods. This demand for safer food percolated up in the value chain and therefore, farmers considering both their and their workers' health and the health of the consumers foray into organic agriculture. However, the shift to organic agriculture is not an easy task in itself and involves costly changes and this shift can be profitable only if there is a premium for the products. Since the local consumers are not capable of purchasing the products with such premium prices, the suppliers prefer catering to the international market. This resulted in organic farming being concentrated in the export-oriented products in Kerala; primarily pepper and coffee. Within the organic food produce industry, there are several types of supply chain. Figure 1 illustrates a generic model of an organic food supply chain existing within the research context and consisting of several tiers illustrated as under:

- $\quad$ Tier 1 - The suppliers to the farmer and within the context of organic food supply chains will include both the raw material suppliers and 'suppliers of knowledge'. In terms of the preferred raw material suppliers, the farmers suggested that they preferred suppliers that had specific characteristics including small and medium 
suppliers, cooperative unions or service societies, governmental divisions that included government endorsed suppliers and universities. Within the organic sector in the research context, the farmer suppliers supply raw materials including bio-fertilizers, biopesticides and seed/sapling suppliers. Since a majority of the players were small and medium farmers, they preferred their suppliers to be similar to them. The raw materials included bio-fertilizers produced through biologically safe and sustainable processes like vermin-culture,composts. Within the context of organic food supply chains, the farmers have to be trained on effective and sustainable methods of farming and the 'suppliers of knowledge' train the farmers accordingly.

- $\quad$ Tier 2 - This tier includes the farmers of fresh produce and they are considered as the custodians of the land. Within the research context, this included only organic farmers who cultivated organic or sustainably grown produce. A significant feature of this industry in the state is the ambiguous definition of organic produce. A majority of the farmers interviewed marketed their produce as 'organic' but on scrutiny, they were not fully organic and were not certified. Such farmers who marketed their products as organic defined themselves as natural farmers (farmers having practices with noninclusion of artificial stimulants like artificial pesticides and fertilizers for farming), GAP farmers (farmers who employed Good Agricultural Practices) and fresh farmers (farmers who sold only locally and sold their products fresh to the consumers). Though such farmers were common, there were a majority of organic farmers; groups ofsmaller farmers who were certified through group certification schemes or individually certified large farmers.

- $\quad$ Tier 3 - This tier consists of traders, service societies and exporting companies that are in charge of supplying to the final business customers. They procure the products from the farmers and they sell it to the retailing organizations and as such,final business customers- businesses, which sell to the consumers. During this tier, there might be processing of the product as in the context of this research, the supply chain consisted of small and medium farmers who lacked the capacity to process the product. The service societies include both for-profit and not-for-profit organizations that are farmer-led cooperative societies that pool resources, knowledge and production.

Tier 4 - The final tier of the supply chain involves the final business customers like that of retailing organizations of organic products. Within the supply chain networks studied in this research, this tier includes both local and international retailers. These retailers sell the products to the end consumers. Though there are many retailers that sell both conventional and organically grown produce, the focus of this research was on retailers selling only organically branded food includingorganic certified, GAP or natural food products.

\subsection{Ethical Rationale for organic agriculture}

The overarching principles of the organic FSC actors mandate operating ethically. Within the context of this research, this means conducting business such that the business upholds the health and safety of not only the soil, but also human beings and the animals that are connected with the operations of this industry. As illustrated prior, there are different supply chain actors and the authors grouped these actors into 
different tiers since they have similar operations and the study illustrates that they also possess similar ethical perceptions - attitudes towards environmental sustainability and social responsibility. The next sections detail the individual tiers' perception of sustainability and social responsibility and thereby illustrate business stakeholders' ethical perceptions.

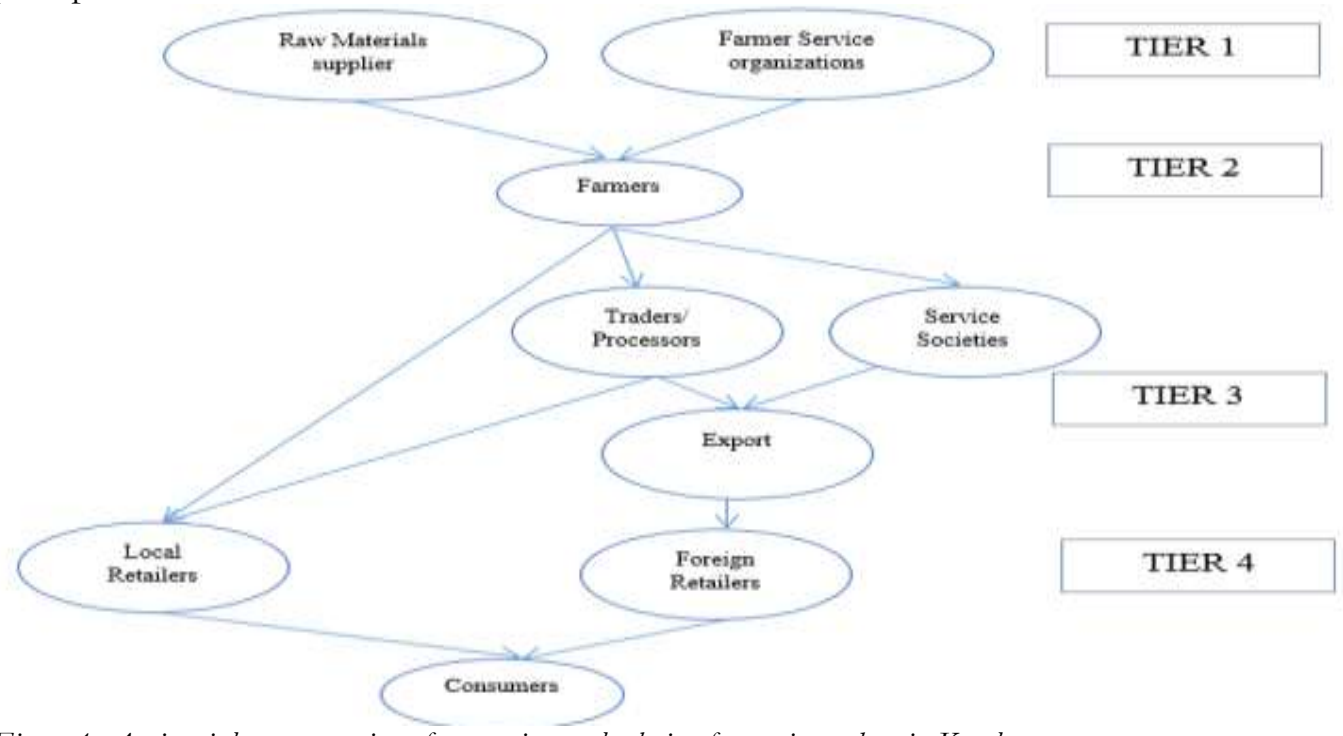

Figure 1. A pictorial representation of a generic supply chain of organic produce in Kerala

\subsection{Stakeholder perception of responsible behaviour}

Tier 1 - Within organic FSC networks and in this research context involving a majority of farmers with small land holdings, it is imperative that there be effective capacity building services. Capacity building involves developing the farming community through the delivery of training programs that will result in better productivity and higher sustainability. The farm service providers deliver such training programs with the idea of capacity building and this is in line with these stakeholders' ethical perceptions. The rationale of the service providers for conducting such programmes is rooted in social responsibility. These service providers want to develop the farmers such that they are self-sufficient thereby alleviating poverty among such farming communities. By implementing such training programs, the service providers will improve farmer capacity and thereby increase resource utilisation and production, thereby resulting in improving the farming community welfare.

Tier 2 -The interview results illustrate that the primary reason for shifting to organic farming was the health of the soil and this, according to the farmer, affects the health of the human beings too. An organic farmer stated his rationale by exemplifying his experience. The harvesting of the produce is usually conducted by poor labour hands; both male and female. The spraying of pesticides and using artificial methods of farming destroys the soil and the immediate environment thereby rendering it hazardous to all animals. In his experience, the children of the labourers and even pregnant women, involved with the farming processes, are commonly seen on the farm for extended periods. This exposure to the "poisonous" soil affected the health of the children. The farmers opined that this interaction with harmful pesticides and fertilizers adversely affected the health of the society; thereby illustrating his motivation to convert to organic farming. The farmers, due to their experience in agriculture stated that their conversion to organic 
farming aimed at "bringing the land back to its pristine non-poisonous state". To quote a farmer when questioned about the ethical underpinning of this industry, he stated "Well.Right now, we have many health related issues. In almost all families, there are members who have major diseases like diabetes and cancer and this is increasing every day. The food we consume is poisonous due to pesticides and ideally only a bealthy individual will be able to sustain his family and thereby, the society. Similarly other farmers and farmer groups suggested that not only did organic farming have its underpinning in sustainability and social responsibility, but also both sustainability and social responsibility were interlinked with each other in this industry.

Tier 3 -Not-for-Profit organizations including service societies, traders and exporting organizations have a different perspective on ethics. The interview results from both the nonprofit and for-profit organizations illustrate the safety and quality of the produce pivotal and this was, according to these actors related to both environmental sustainability and social responsibility. Since the produce, usually transported from neighbouring states are perceived to be unhealthy, responsible behaviour, according to such stakeholders will involve dealing in and selling the most safe and healthy products; safe for both the environment, and consumers and the agriculturists. The for-profit organizations considered both the environment and the stakeholder health to be important but more from a business perspective. This meant that they accounted for fairness in operations and fair distribution of fiscal returns as important as the environmental sustainability. The interview results illustrate that these players (both for-profit and not-forprofits) created systems that increased efficiency of transportation and revenue distribution thereby facilitating the business processes.

Tier 4 - For the foreign retailers and final business customers based overseas, the ethical underpinning of the industry was based ongreater fairness to the upstream supply chain actors, mainly the farmers. The essence of the product and the industry, according to these actors lay in equitable business practices and the non-opportunistic behaviour towards the farmers. The adherence to fair principles, according to these actors, willresult in the emancipation of the poorer farmer and such ideologies represent the social responsibility of the business. They considered the organic product, as safe and healthy for their consumers. Similarly, for local retailers too, the ethical underpinning affecting the responsibility orientation of the retailers is based on consumer health and the equitable business of the farmers. Furthermore, the interviewed local retailers emphasized the importance of consuming healthy products and they wanted such food products to be accessible to the public at affordable prices. Affordable prices, as the retailers suggested, must not be at the expense of farmer's profitability and, therefore, they mandated fair remuneration to the farmers too.

\section{Discussion}

A significant difference from other stakeholders was that the final business customers were more concerned about the social influence of the industry and less concerned about the environmental sustainability of the organic industry. In comparison to other stakeholders, including the farmers and the tier 3 actors, the final business customers were more interested in the quality of the product; they mandate safe and healthy products with fair remuneration for other actors along the supply chain, especially the farmer. From Tier 1 to Tier 4, there is a gradual decrease in the importance associated with environmental sustainability and correspondingly, an increase in the importance associated with the social welfare of the stakeholders of this industry. The final business customers including the local and international retailers were more interested in giving fair returns to the farmers and the delivery of safe and healthy 
products to the consumers; thereby illustrating importance of social welfare of the industry. Though they considered the environmental sustainability as a prerequisite for the organic product, the results illustrate that the final business customers (tier 4) considered the social aspect of the business most important. In comparison to the same, the farmers (tier 2) and the stakeholders directly associating with the farmers (tier 1 and tier 3) prioritised environmental sustainability over social responsibility. Notwithstanding this, these tiers considered social responsibility as important for the industry and this is implied through their importance associated with the health of the farmers and the stakeholders within the farming community.

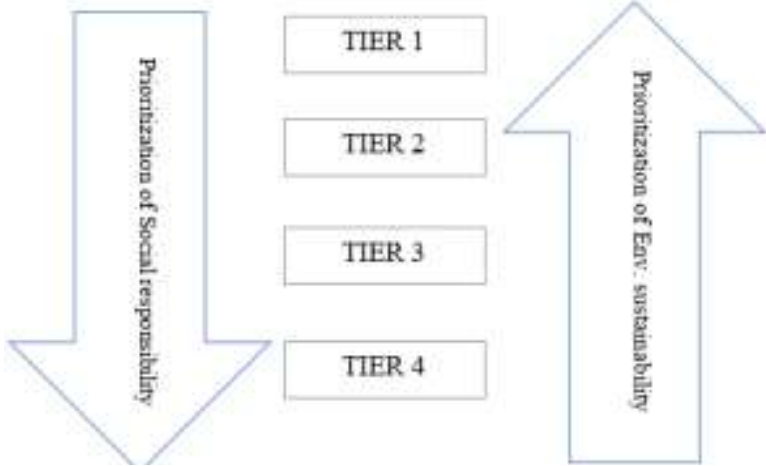

Figure 2. Differences in prioritization of environmental sustainability and social responsibility along the supply chain

Explicating this in detail - it can be stated that the cause for a shift in priority is the relative role and stakeholders that these players associate with. To exemplify the same, the paper considers the case of a farmer. The farmer directly interacts with the soil and the immediate environment and stakeholders like the labour hands, the children of these labourers and organisms that affect the product and soil quality like that of earthworms and pollination agents. Incessant use of artificial fertilizers affects the quality of the soil and farm friendly animals adversely and the farmers witness this "firsthand". Therefore, the farmer's rationale and ethical underpinning influences them to be more environmentally sustainable, thereby not affecting the health of the soil and such stakeholders (including farm friendly animals) negatively. Thus, they opt for more sustainable methods of agriculture and prioritise environmental sustainability. In contrast, the retailers associate with the end consumers of the products and witness health issues associated with consuming non-healthy and unsafe food. Thus, their role and association with the consumers influence their ethical perceptions. Makatouni (2002) illustrates that from a consumer's perspective, the values pertaining to the human being is the most important and only then does animal-centric values and environment centric values become priority. Similarly, from a consumer perspective, as Zander and Hamm (2010) suggests -the ethical attributes of the organic consumers is affected by welfare of the animals and farmers and the "localness" of the product. The localness pertains to the distance between the point of production and point of consumption and the lower this distance is, the better it is. Other ethical attributes affecting consumer decisions included price of the product, bio-diversity, social criteria, care of the farm and cultural features 
(Zander \& Hamm, 2010). This research by Zander and Hamm (2010) was within the European context and since the organic products produced in Kerala was primarily aimed for the Western market (due to their ability to give a premium price), the reason for a shift in values from sustainability to social responsibility can be stated to be due to the market preferences. This mandates the tier 3 supply chain actors, within the Kerala research context, to supply their consumers with safe and healthy products, thereby increasing the health of the foreign consumers and this makes them prioritise social responsibility over environmental sustainability.

An analysis of the organic industry's values, illustratethese values as being based on environmental sustainability and social responsibility. This research, by understanding the ethical perceptions of all supply chain actors, states that these values do indeed drive the industry since such values affect the ethical perceptions of all supply chain actors. Conventionalisation has had its effect on the organic industry as is illustrated by the addition of some artificial inputs in this industry. The products cultivated by these methods, though not certified as organic, are marketed under the organic banner. However, even though this has an effect on the environmental sustainability of the industry, they still follow Good Agricultural Practices and adhere to natural farming systems. This increases the environmental sustainability of the industry and thereby contributes to the ethical underpinning of the industry.

\section{Limitations and directions for future research}

The generalizability of this research could be limited due to its qualitative nature. Furthermore, the context of this research is within the South Indian context and the researchers predominantly interviewed organic stakeholders within the South Indian state of Kerala, a state that has limited agriculture compared to other states in India. A significant characteristic of the residents of Kerala is that they are highly educated and in comparison to the other states, the farmers and other organic FSC actors are more educated. Since education can affect their perception of ethics, this might affect the generalizability of these findings, since the FSC, stakeholders in other states are less educated and thereby less knowledgeable. This paper illustrates that there is a difference in perception of ethics among different stakeholder groups and the paper explores such differences in ethical perception along different supply chain stakeholders. Since such comprehensive research along different supply chain levels is non-existent within this industry, the results of this paper can guide future research within the global market.

\section{Conclusion}

The organic industry, having its ideology deeply rooted in the ethical conduction of business through environmentally sustainable and socially responsible operations, is subject to critique due to greater conventionalisation of the industry. Therefore, such a study of the ethical underpinning of an entire industry was mandatory to ascertain the importance associated with sustainability and responsibility by all the business stakeholders within the industry. Within the organic food network existing in Kerala, there are several players and based on their relative role within the supply chain, the 
perception of ethics change. As the paper explains, there is a gradual change in the prioritisation of environmental and social responsibility but the industry still considers environmental sustainability and social responsibility as its driving forces.

\section{References}

Chandrashekar, H. (2010). Changing scenario of organic farming in India: An overview. International NGO Journal, 5(2), 034-039.

Darnhofer, I., Lindenthal, T., Bartel-Kratochvil, R., \& Zollitsch, W. (2010). Conventionalisation of organic farming practices: from structural criteria towards an assessment based on organic principles. A review. Agronomy for Sustainable Development, 30(1), 67-81.

De Wit, J., \& Verhoog, H. (2007). Organic values and the conventionalization of organic agriculture. NJASwageningen journal of life sciences, 54(4), 449-462.

Duram, L. A. (1999). Factors in organic farmers' decisionmaking: diversity, challenge, and obstacles. American Journal of Alternative Agriculture, 14, 2-10.

Filipe, Santos, K., \& Eisenhardt, M. (2004). Multiple Case Study. The SAGE Encyclopedia of Social Science Research Methods. Sage Publications, Inc. Thousand Oaks, CA: Sage Publications, Inc.

Freyer, B., Bingen, J., Klimek, M., \& Paxton, R. (2015). Feeding the World-The Contribution of IFOAM Principles Re-Thinking Organic Food and Farming in a Changing World (pp. 81-102): Springer.

Gadzikwa, L., Lyne, M., \& Hendriks, S. (2006). Collective action in smallholder organic farming: a study of the ezemvelo farmers'organization in kwazulu-natal. South African journal of economics, 74(2), 344358.

Glasbergen, P. (2011). Mechanisms of private meta-governance: an analysis of global private governance for sustainable development. International Journal of Strategic Business Alliances, 2(3), 189-206. doi: 10.1504/IJSBA.2011.040886

Honkanen, P., Verplanken, B., \& Olsen, S. O. (2006). Ethical values and motives driving organic food choice. Journal of Consumer Behaviour, 5(5), 420-430.

Jeromi, P. D. (2007). Farmers' Indebtedness and Suicides: Impact of Agricultural Trade Liberalisation in Kerala. Economic and Political Weekly, 42(31), 3241-3247. doi: 10.2307/4419874

Klonsky, K. (2012). Comparison of Production Costs and Resource Use for Organic and Conventional Production Systems. American Journal of Agricultural Economics, 94(2), 314-321.

Lamine, C., \& Bellon, S. (2009). Conversion to organic farming: a multidimensional research object at the crossroads of agricultural and social sciences. A review. Agronomy for Sustainable Development, 29(1), 97-112. doi: 10.1051/agro:2008007

Lea, E., \& Worsley, T. (2005). Australians' organic food beliefs, demographics and values. British Food Journal, $107(11), 855-869$.

Macharia, J., Collins, R., \& Sun, T. (2013). Value-based consumer segmentation: the key to sustainable agrifood chains. British Food Journal, 115(9), 1313-1328.

Makatouni, A. (2002). What motivates consumers to buy organic food in the UK?: Results from a qualitative study. British Food Journal, 104(3/4/5), 345-352.

Sligh, M., \& Cierpka, T. (2007). Organic values (pp. 30-39). Wallingford: CABI.

Stockdale, E., Lampkin, N., Hovi, M., Keatinge, R., Lennartsson, E., Macdonald, D., . . Watson, C. (2001). Agronomic and environmental implications of organic farming systems. Advances in Agronomy, 70, 261-327.

Stolze, M., \& Lampkin, N. (2009). Policy for organic farming: Rationale and concepts. Food Policy, 34(3), $237-$ 244.

Zainal, Z. (2007). Case study as a research method. Jurnal Kemanusiaan(9), 1-6.

Zander, K., \& Hamm, U. (2010). Consumer preferences for additional ethical attributes of organic food. Food quality and preference, 21(5), 495-503. 